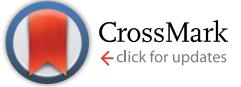

Cite this: RSC Adv., 2017, 7, 12990

Received 6th December 2016 Accepted 17th February 2017

DOI: 10.1039/c6ra27842f

rsc.li/rsc-advances

\section{Fast direct detection of natural dyes in historic and prehistoric textiles by flowprobe ${ }^{\text {TM}}$-ESI-HRMS $\uparrow$}

\author{
Annemarie E. Kramell, ${ }^{a}$ Alexander O. Brachmann, ${ }^{b}$ Ralph Kluge, ${ }^{a}$ Jörn Piel $^{\mathrm{b}}$ \\ and René Csuk*a
}

Dyestuff analyses were performed directly from the surface of different bluish and reddish colored historic textile samples by flowprobe ${ }^{T M}$-electrospray ionization-high-resolution mass spectrometry (flowprobe ${ }^{T M}$ ESI-HRMS). This real-time in situ microextraction method allowed rapid, reliable and minimal-destructive analysis without extra and time-consuming sample preparation and required only a minimum amount of valuable archaeological material. As demonstrated for indigo-type and anthraquinone dyes this technique is useful for the analysis of various types of textiles regardless of their fiber matrix, appearance or handicraft and is also suitable for investigating fragile archeological fibers. Thus, flowprobe ${ }^{T M}$-ESIHRMS is a promising analytical tool for characterizing organic colorants in objects of archaeological interest.

\section{Introduction}

Historic and prehistoric textiles are among the most precious but also most rare artifacts of ancient cultures - often long gone, neglected and even forgotten. Knowledge about these cultures and their daily lifestyle seems important to understand historic developments, progress in science and technology but also to comprehend why ancient cultures sometimes vanished. Usually, the number and amount of available samples are small, and special ways of investigation are called for. Therefore, mass spectrometry (MS) is unquestionably one of the most powerful tools in modern archaeometry ${ }^{\mathbf{1 , 2}}$ to examine the artifacts from archaeological excavations and finding sites. In this context, investigating natural dyes in historic textiles is one of the central parts of this research. The identification of colorants is essential for the conservation and restauration of these objects. In addition, information about colorants help to understand ancient technologies and cultural exchanges. They also help answering questions concerning the social status of the bearers of clothes. ${ }^{3}$

Our ancestors used a wide range of organic dyestuffs for coloring different fiber materials. ${ }^{4-6}$ Therefore, techniques used to characterize dyestuffs should enable the detection of organic

\footnotetext{
${ }^{a}$ Department of Organic Chemistry, Martin-Luther-University Halle-Wittenberg, Kurt-Mothes-Strasse 2, 06120 Halle, Germany.E-mail: rene.csuk@chemie.uni-halle.de ${ }^{b}$ Institute of Microbiology, Eidgenössische Technische Hochschule (ETH) Zürich, Vladimir-Prelog-Weg 1-5, 8093 Zürich, Switzerland

$\dagger$ Electronic supplementary information (ESI) available: Fig. S1-S7 and Table S1 showing schematic illustration of a junction region from a flowprobe ${ }^{\mathrm{TM}}$-MS experimental setup, further MS spectra, investigated historic samples and detailed information regarding the characterization of dyestuffs. See DOI: $10.1039 / \mathrm{c} 6 \mathrm{ra} 27842 \mathrm{f}$
}

molecules of different dyestuff classes regardless of the used fiber material or the properties of the sample. Detecting dyes in historic textiles is a difficult problem, because archaeologic textiles are unique, precious and often very fragile. Usually only very small samples are provided for different analytic purposes (e.g. fiber analysis, ${ }^{14} \mathrm{C}$-dating, dyestuff analysis). Besides, the dye components form only a small fraction of the mass of the sample as compared to the mass of the fibers. Thus, the challenging task is to detect an analyte in low concentration in a small sample if possible without destroying the sample. Also, the conservation status of the historic textiles plays an important role, because different chemical, photochemical or microbiological aging complicate detecting the dyestuff.

First references and conclusions concerning the colorants can be obtained from minimal- or non-destructive spectroscopic techniques such as visible reflectance, Raman or ATR-IR spectroscopy. ${ }^{7-9}$ Nonetheless, signals originating from fiber materials, degradation products etc. often also contribute to the measuring signal, and resulting interferences complicate the identification of coloring materials. In addition, natural dyes are usually a mixture of structurally similar compounds. However, in situ spectroscopic methods are of only limited value for the characterization of mixtures of dyestuffs or unknown dye components.

In the past decades chromatographic methods, e.g. (U)HPLCDAD [(ultra) high performance liquid chromatography -diode array detection], (U)HPLC-ESI-MS/MS [(U)HPLC-electrospray ionization tandem mass spectrometry] or HPLC-APCI-MS/MS (HPLC-atmospheric pressure chemical ionization tandem mass spectrometry) were established as standard methods for organic dyestuff analysis; all of them require a previous extraction of the dyes from the fibers. ${ }^{\mathbf{1 0 - 1 7}}$ Usually, these 
extractions are a time-consuming preparation step, and rather harsh conditions (for example, treating with hydrochloric acid at $100{ }^{\circ} \mathrm{C}$ (ref. 18)) are necessary to remove the dyes from the fibers. Occasionally it is even necessary to combine different extraction methods to get an optimal result, especially when the coloration consists of different classes of dyestuff. However, extraction steps are often limited by the available amount of the archaeological samples, and harsh extraction conditions may result in a decomposition of the dyes or lead to structural changes of sensitive dyes. For example, using hydrochloric acid has been shown to cause structural changes for the neoflavonoids hematein and brazilein. ${ }^{18}$

Nowadays, techniques based on direct mass spectrometry such as LDI (laser desorption/ionization)-MS, ${ }^{19}$ TOF-SIMS (timeof-flight secondary ion mass spectrometry), ${ }^{9,20}$ DART (direct analysis in real time)-MS $\mathbf{M S}^{\mathbf{2 1 , 2 2}}$ and ASAP (atmospheric solids analysis probe)-MS ${ }^{23}$ become popular. These methods allow the analysis of dyestuffs directly from the archaeological fiber. They need only small amounts of sample and avoid the disadvantages of the above mentioned extractive methods. Interestingly, the use of direct MS methods including in situ extractions, for example DESI (desorption electrospray ionization)- and LMJSSP (liquid microjunction surface sampling probe)-MS, for dyestuff analyses of ancient textiles was until now not reported, although these methods were successfully applied in biochemical and forensic research. ${ }^{24-31}$

In this study, we present the first rapid and minimally invasive dyestuff analysis under ambient conditions through real-time in situ microextractions of fibers by flowprobe ${ }^{\mathrm{TM}}$-ESIHRMS (high-resolution MS), a commercial LMJ-SSP device coupled with an Orbitrap mass analyzer. This system uses a pair of coaxial capillaries (probe outer diameter $=630 \mu \mathrm{m}$ ) being positioned close to the surface of the examined object. A continuous solvent flow forms a liquid microjunction between the probe and the sample surface and delivers the extractable analytes to the ESI mass spectrometer (for a detailed description, see (ref. 27) and Fig. S1†). Thus, dyestuff analyses were performed directly from bluish and reddish colored archaeological fibers. The studied samples consisted of wool, silk and cotton and have been colored with indigo-type and anthraquinone dyes; these colorants represent the most important "ancient" natural organic dyestuffs to create blue and red hues. The archaeological objects of this investigation originated from different cultures and continents and cover a time span from 1st millennium BC to the 15 th or 16 th century. Thus, the historic fibers differed in material, age, conservation status and workmanship.

\section{Results and discussion}

In a recent study we applied ASAP-MS ${ }^{23}$ for the direct characterization of indigoid dyes in various historic textiles. However, rather harsh conditions of the ionization process were unsuitable for detecting (quasi)molecular ions of more sensitive colorants, e.g. carminic acid. In these cases only fragment ions were observed which did not allow the unequivocal identification of the dyes. Compared to ASAP-MS, DESI-MS and flowprobe ${ }^{\mathrm{TM}}$-ESI-MS use significantly milder ionization conditions (for a comparison of the ASAP- and flowprobe ${ }^{\text {TM-ESI-MS }}$ spectra, see Fig. $\mathrm{S} 2 \dagger$ ). Therefore, both methods were tested for detecting indigoid and anthraquinone dyes on textile fibers. Surprisingly, no stable signals of the colorants were observed in the DESI-MS experiments, while the flowprobe ${ }^{\text {TM}}$-ESI-MS method turned out to be the method of choice for the detection of both dyestuff types (see below).

\subsection{In situ microextraction via flowprobe ${ }^{\mathrm{TM}}$ HRMS}

In a first step, the ionizability and extractability of genuine standard compounds (different indigo-type and anthraquinone colorants) was tested. The experimental setup was optimized, followed by a thoroughly investigation of reference fibers [undyed wool, silk and linen followed by fibers dyed with synthetic indigo, alizarin, purpurin, woad (Isatis tinctoria L.), madder (Rubia tinctorum L.) or with food colorant E120 (extract of the scale insect Dactylopius coccus Costa)]. Finally, bunches of fibers or tiny yarn fragments of red and blue colored archaeological textiles originating from ancient Peru and China (Table 1, samples A-G) were analyzed.

The investigations were performed without any extra sample preparation; thus, the fibers were simply fixed on a conventional glass slide by wetting with a drop of solvent, and characteristic dyestuff signals could be detected within a few seconds (Fig. S3 $\dagger$ ). The extraction was monitored over a period of about 2 minutes for enhanced signal-to-noise and to prevent any discrimination of compounds with a poorer extractability. Thus, the complete analysis including sample preparation and measuring takes less than 5 minutes which is in contrast to the time-consuming extractions (LC-MS). ${ }^{32}$

A typical flowprobe ${ }^{\mathrm{TM}}$ HRMS spectrum of red wool fibers dyed with an extract of the scale insect Dactylopius coccus Costa is displayed in Fig. 1. This spectrum shows an intense peak at $m / z 491.0834\left([\mathrm{M}-\mathrm{H}]^{-}\right)$, assigned to the main colorant carminic acid and isomers such as deIV and deVII (dcIV: 7-C- $\alpha$-D-glucofuranoside of kermesic acid; dcVII: 7-C- $\beta$-D-glucofuranoside of kermesic acid ${ }^{\mathbf{4 1 , 4 2}}$ ). Also, minor components of the cochineal dye were detected with lower intensities, e.g. kermesic acid, flavokermesic acid and dcII-like compounds (dcII is the 7-C- $\alpha-\mathrm{D}^{-}$ glucopyranoside of flavokermesic acid ${ }^{\mathbf{4 1}}$ ).

The dyestuffs detected in the historic samples by flowprobe $^{\mathrm{TM}}$ HRMS are summarized in Table 1 and the respective structures are shown in Scheme 1. Signals of the corresponding (quasi)molecular ions were observed in all cases with high mass accuracy (error $\leq 1.5 \mathrm{ppm}$, Table $\mathrm{S} 1 \dagger$ ). The composition of the detected compounds allowed easy determination of the dye sources (plants or scale insects) used for the coloration of the ancient textiles (see below). Furthermore, the results were confirmed by HPLC-MS/MS and HPLC-DAD investigations, which additionally enabled the identification of isomers by comparing the spectra and retention times with those of authentic reference compounds (Table 1).

For example, HRMS spectra of the red-shaded historic samples A, E (Fig. 2) and F were similar to those of reference fibers dyed with madder and were dominated by signals of 


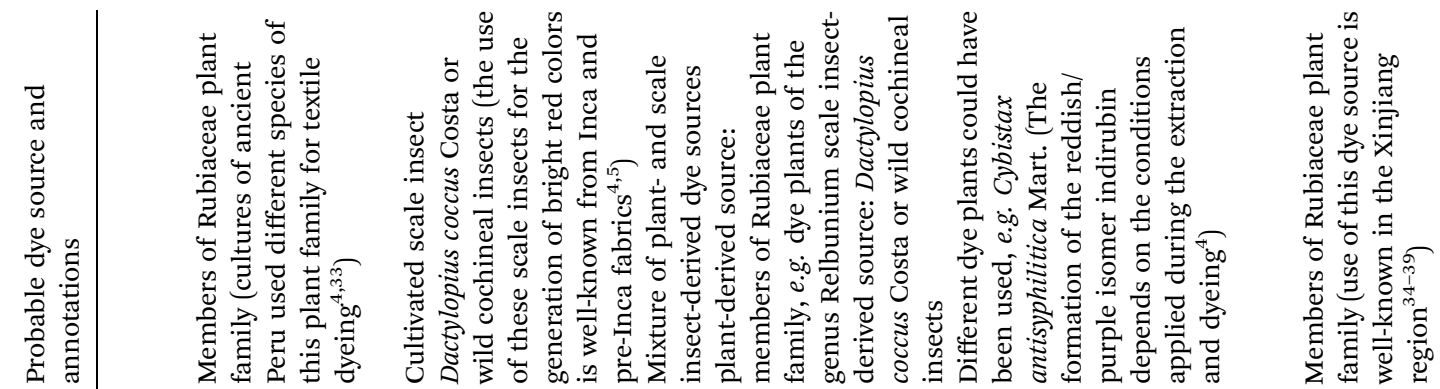

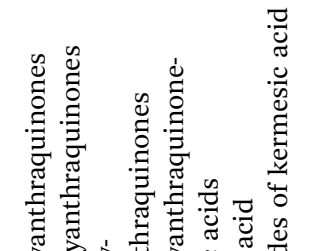

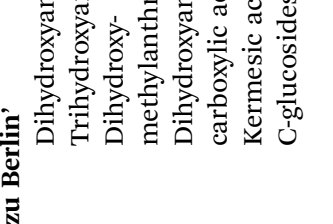

㟧

苟

$\bar{\circ}$

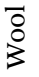
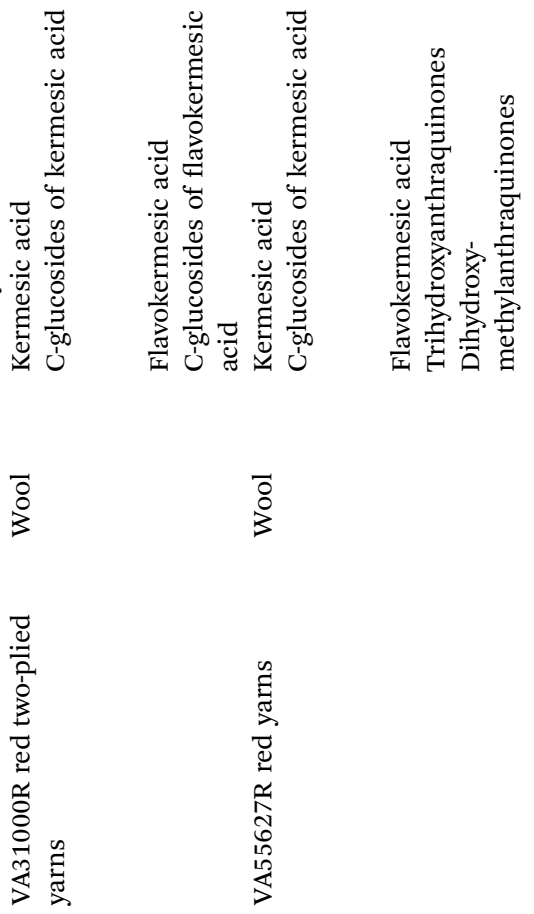

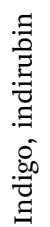
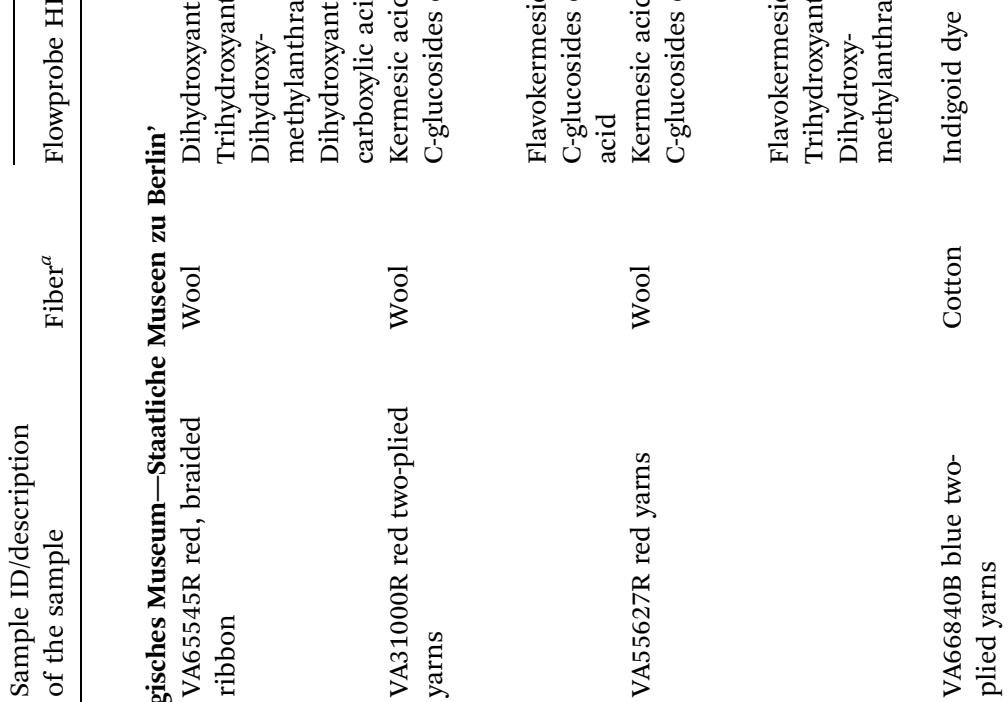

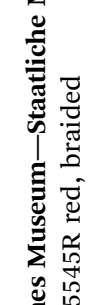

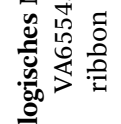

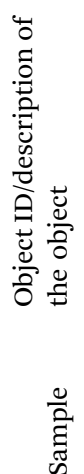
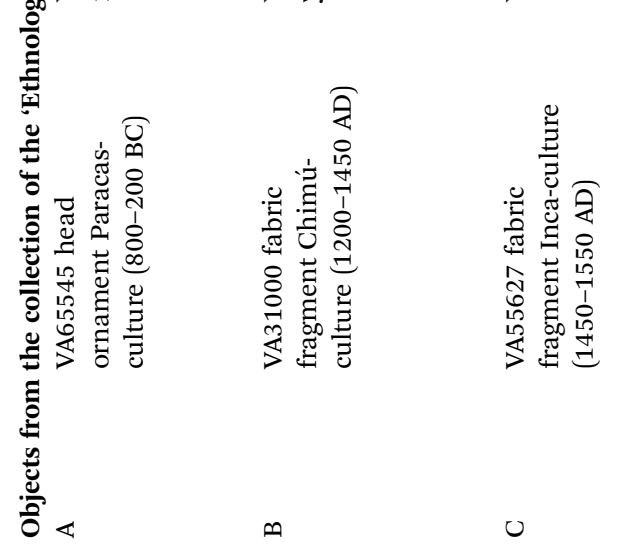
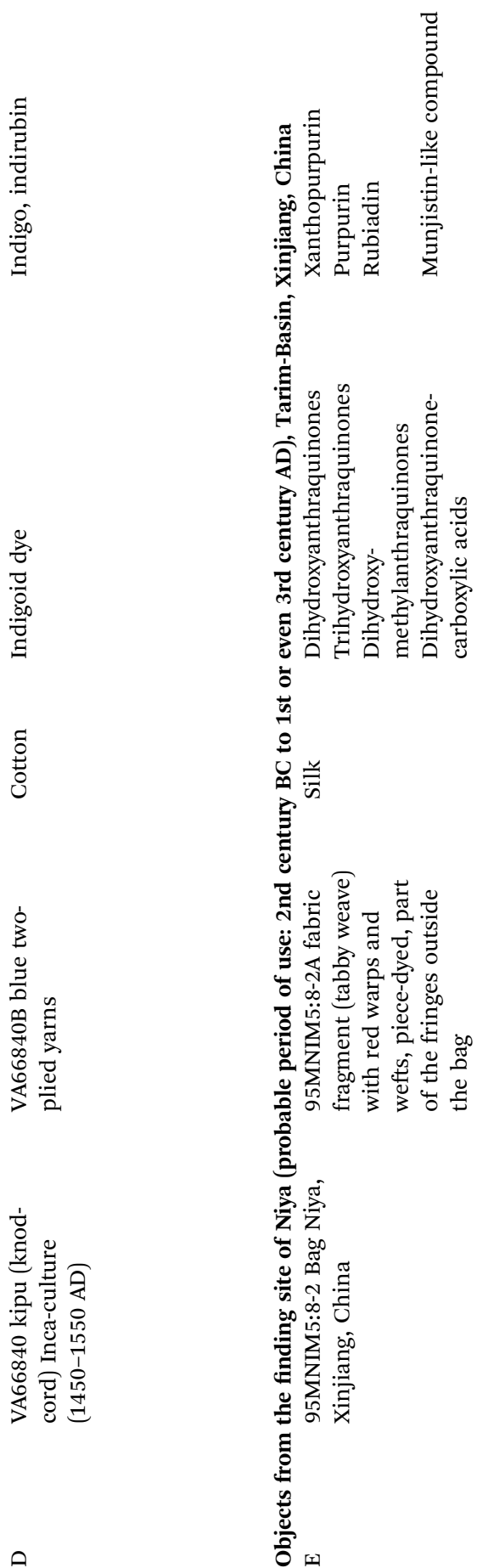


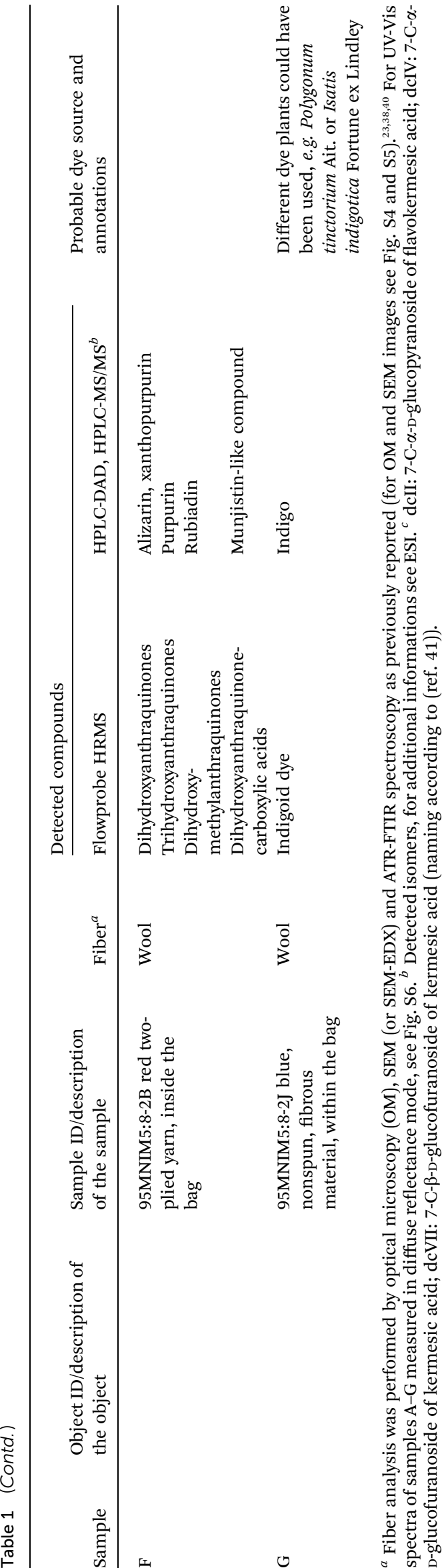

dihydroxyanthraquinones, trihydroxyanthraquinones and dihydroxy-methyl-anthraquinones, which were identified by HPLC as alizarin, xanthopurpurin, purpurin and rubiadin. And, an extra intense signal at $m / z 283.0248$ was observed, which points to the presence of a dihydroxyanthraquinone-carboxylic acid. Due to the high resolution data alternative structures, e.g. physcion (molecular formula: $\mathrm{C}_{16} \mathrm{H}_{12} \mathrm{O}_{5}$ ) or mollugin (molecular formula: $\mathrm{C}_{17} \mathrm{H}_{16} \mathrm{O}_{4}$ ), detected in some Rubia species $^{43,44}$ can be excluded. The present compound, most probably an until now unknown isomer of munjistin (1,3dihydroxyanthraquinone-2-carboxylic acid), has been already detected by LC-ESI-MS/MS in several samples - including samples E and F - from the finding site of Niya (archaeological site in Xinjiang, China). ${ }^{45}$ Dihydroxyanthraquinones, trihydroxyanthraquinones and dihydroxy-methyl-anthraquinones are characteristic dyes from plants of the Rubiaceae family. Plants from this family are found globally, and especially some genera (e.g. Rubia, Galium and Relbunium) have been used in many cultures for obtaining red hued textiles. ${ }^{37,46-48}$

In contrast, signals for carminic acid, its isomers, for flavokermesic acid and kermesic acid were observed in spectra of the red-shaded historic samples C and B. A peak of dcII-like compounds at $m / z 475.0881$ was only detected on fibers of sample B. The presence of these anthraquinone dyestuffs is in agreement with the use of cochineal insects for coloring in ancient Peruvian cultures. ${ }^{4}$ Interestingly, spectra of sample C showed extra peaks at $\mathrm{m} / \mathrm{z} 253.0506$ and $\mathrm{m} / \mathrm{z} 255.0299$, corresponding to quasimolecular ions $\left([\mathrm{M}-\mathrm{H}]^{-}\right)$of anthraquinones identified as rubiadin and purpurin. These anthraquinones are not components of a scale insect dye, suggesting the additional use of a plant-derived colorant (for example a Relbunium species). It is worth mentioning that it is possible to associate the utilization of cochineal and plant-derived colorants as red dye sources with certain cultural periods (from $300 \mathrm{BC}$ to the 16th century AD) of the Pre-Columbian Peru. Going from the earlier cultures (e.g. Paracas and Nazca) to the later (e.g. Chimu, Chancay and Inca) one observes the gradual replacement of vegetal dye sources such as Relbunium by conchineal. ${ }^{49}$

For all blue-colored references (fibers dyed with synthetic indigo or woad) the quasimolecular ion $[\mathrm{M}+\mathrm{H}]^{+}$at $\mathrm{m} / \mathrm{z}$ 263.0815, assigned to indigo-type dyes like indigo and indirubin, was detected. A signal of indigo-type dyes at $\mathrm{m} / \mathrm{z} 263.0815$ was also observed for the blue historical samples D and G.

In summary we can note that the LMJ-SSP-HRMS experiments clearly revealed the use of a cochineal dye and of a dye gained from plants of the Rubiaceae family for the generation of the investigated red hues and the use of the very important dye indigo for blue colorations. The confirmation of dye sources was successful regardless of the fiber materials (silk, wool or cotton), the age of the sample, or the sample's appearance or handicraft. A critical remark should be mentioned, which, however, was not relevant for the identification of the dye sources in our case. The identification and assignment of isomeric structures by LMJ-MS technology (as with all other direct MS methods) is possible only to a limited extent by an interpretation of the fragmentation pattern. This problem can be solved by incorporation of an LC 


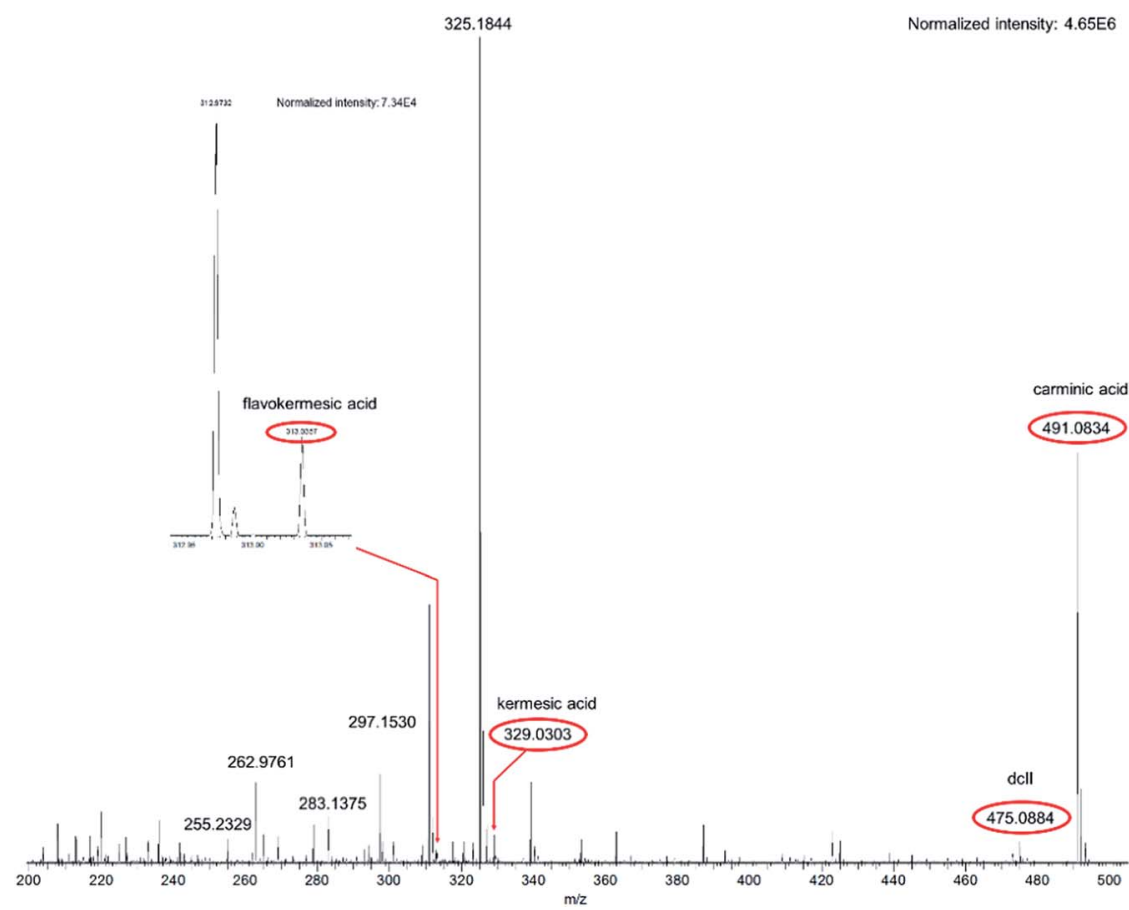

Fig. 1 FlowprobeTM HRMS spectrum of woolen reference fibers dyed with an extract of the scale insect Dactylopius coccus Costa. MS detection of the anthraquinones was performed in negative ion full-scan mode, and the marked substances were detected as quasimolecular ions $\left([\mathrm{M}-\mathrm{H}]^{-}\right)$.

system between the surface sampling device and the mass spectrometer, as described by Kertesz and Van Berkel for the separation of isomeric drug metabolites. ${ }^{50}$

Indigo-type dyes
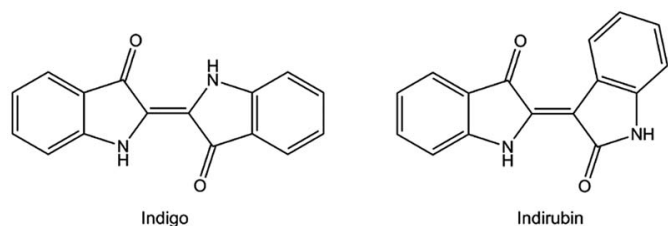

Anthraquinone dyes<smiles>[R]c1cc2c(c([R])c1[R])C(=O)c1c(O)c([R])c([R])c([R])c1C2=O</smiles>

\begin{tabular}{|c|c|c|c|c|c|c|}
\hline Substance & $\mathbf{R}^{1}$ & $\mathbf{R}^{2}$ & $R^{3}$ & $\mathbf{R}^{4}$ & $\mathbf{R}^{5}$ & $\mathrm{R}^{6}$ \\
\hline Alizarin & $\mathrm{H}$ & $\mathrm{H}$ & H & $\mathrm{H}$ & H & $\mathrm{OH}$ \\
\hline Carminic acid & $\mathrm{CH}_{3}$ & $\mathrm{COOH}$ & $\mathrm{OH}$ & $\mathrm{OH}$ & $\mathrm{OH}$ & C-a-D-Glucopyranosyl \\
\hline dcll & $\mathrm{CH}_{3}$ & $\mathrm{COOH}$ & $\mathrm{OH}$ & $\mathrm{H}$ & $\mathrm{OH}$ & C-a-D-Glucopyranosy \\
\hline $\begin{array}{c}\mathrm{dclV} \\
\text { (isomer of carminic acid) }\end{array}$ & $\mathrm{CH}_{3}$ & $\mathrm{COOH}$ & $\mathrm{OH}$ & $\mathrm{OH}$ & $\mathrm{OH}$ & C- $a-D$-Glucofuranosyl \\
\hline $\begin{array}{c}\text { dcVII } \\
\text { (isomer of carminic acid) }\end{array}$ & $\mathrm{CH}_{3}$ & $\mathrm{COOH}$ & $\mathrm{OH}$ & $\mathrm{OH}$ & $\mathrm{OH}$ & C- $\beta$-D-Glucofuranosyl \\
\hline Flavokermesic acid & $\mathrm{CH}_{3}$ & $\mathrm{COOH}$ & $\mathrm{OH}$ & $\mathrm{H}$ & $\mathrm{OH}$ & $\mathrm{H}$ \\
\hline Kermesic acid & $\mathrm{CH}_{3}$ & $\mathrm{COOH}$ & $\mathrm{OH}$ & $\mathrm{OH}$ & $\mathrm{OH}$ & $\mathrm{H}$ \\
\hline Munjistin & $\mathrm{H}$ & $\mathrm{H}$ & $\mathrm{H}$ & $\mathrm{H}$ & $\mathrm{OH}$ & $\mathrm{COOH}$ \\
\hline Purpurin & $\mathrm{H}$ & $\mathrm{H}$ & H & $\mathrm{OH}$ & H & $\mathrm{OH}$ \\
\hline Rubiadin & $\mathrm{H}$ & $\mathrm{H}$ & $\mathrm{H}$ & $\mathrm{H}$ & $\mathrm{OH}$ & $\mathrm{CH}_{3}$ \\
\hline Xanthopurpurin & $\mathrm{H}$ & $\mathrm{H}$ & $\mathrm{H}$ & H & $\mathrm{OH}$ & H \\
\hline
\end{tabular}

Scheme 1 Structures of the detected dyestuffs.
One important point especially in the investigations of archaeological material is the question if the sample will be destroyed during the measurement. From Fig. 3 it is obvious that no significant change of color was observed comparing the introduced and the recovered material after the flowprobe ${ }^{\mathrm{TM}}$ analysis (for a further example, see Fig. S7 $\dagger$ ). Thus, the sample can be used for further investigations and the method may be characterized as minimal-destructive.

\section{Experimental}

\subsection{Chemicals and materials}

Indigo and alizarin (97\%) were bought from ACROS Organics; carminic acid $(\geq 96 \%)$ from Fluka Analytical, indirubin $(\geq 98 \%)$ and the aqueous $\mathrm{NH}_{4} \mathrm{OH}$ solution (p.A.) from Sigma-Aldrich, xanthopurpurin from Rare Chemicals $\mathrm{GmbH}(\mathrm{Kiel}), \mathrm{KAl}\left(\mathrm{SO}_{4}\right)_{2}{ }^{-}$ $.12 \mathrm{H}_{2} \mathrm{O}$ (puriss.) from Riedel-de Haën and potassium bitartrate (krist. DAB. 6) from VEB Laborchemie APOLDA. Purpurin has been obtained from Aldrich's collection of rare chemicals. A mixture consisting of kermesic acid and flavokermesic acid as well as a woolen yarn (previously mordant dyed using alum and munjistin) has been obtained from the collection of historical colorants (Technical University of Dresden, Prof. Dr H. Hartmann). Rubiadin has been synthesized according to procedures of Takano et al. ${ }^{51}$ Dried rhizomes from madder (Rubia tinctorum L.) were bought from Kremer Pigmente GmbH \& Co KG (Aichstetten). E120, a food dye [exctract from Cochenille lice (Dactylopius coccus Costa)] was obtained from Sensient Food Colors (Geesthacht). A sheep wool fleece ( $100 \%$ merino wool) and linen fabric ( $59 \%$ linen and $41 \%$ modal) were purchased from Galerie Smend (Köln). Nonspun natural silk (mulberry silk from Bombyx mori L.) and 
Normalized intensity: $6.35 \mathrm{E} 6$

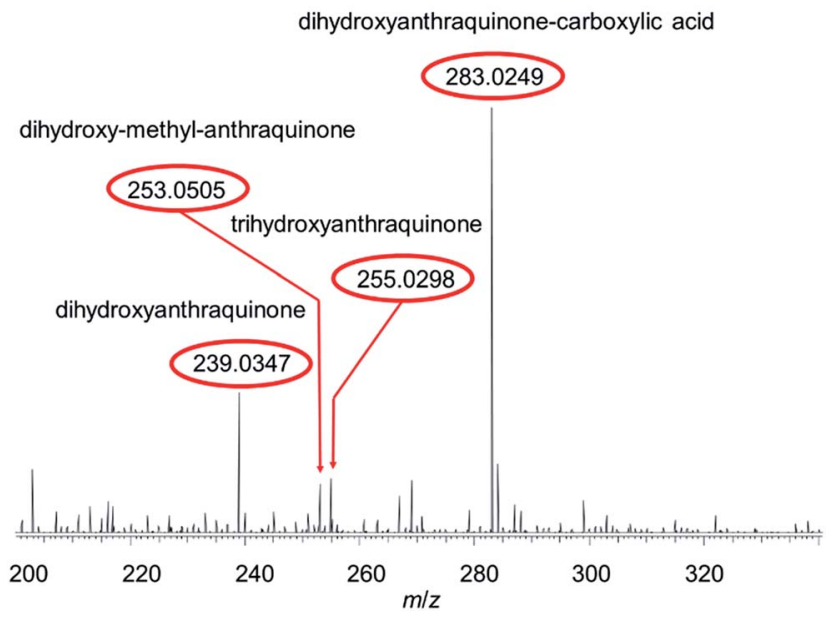

Fig. 2 Flowprobe TM HRMS spectrum of the red shaded historic sample $E$ (silk fabric). MS detection of anthraquinones was performed in negative ion full-scan mode, and the marked substances were detected as quasimolecular ions $\left([\mathrm{M}-\mathrm{H}]^{-}\right)$.

a wool fleece [previously vat dyed using leaves and stems of woad (Isatis tinctoria L.), dyeing according to (ref. 38)] was obtained from a collection of fibers (Landesamt für Denkmalpflege und Archäologie Sachsen-Anhalt - Landesmuseum für Vorgeschichte, by courtesy of Mrs F. Hertel and of Dr C.-H. Wunderlich). A woolen yarn [previously mordant dyed using alum and dried rhizomes from madder (Rubia tinctorum L.)] was obtained from Dr K. Kania, Erlangen. All reference fibers were washed before investigation [distilled water, $40{ }^{\circ} \mathrm{C}$ (for silk) and $80{ }^{\circ} \mathrm{C}$ (for wool and plant derived fibers)], respectively.

\subsection{Instrumentations}

Optical microscopic (transmission light and digital microscope), ATR-FTIR (Fourier transform infrared in attenuated

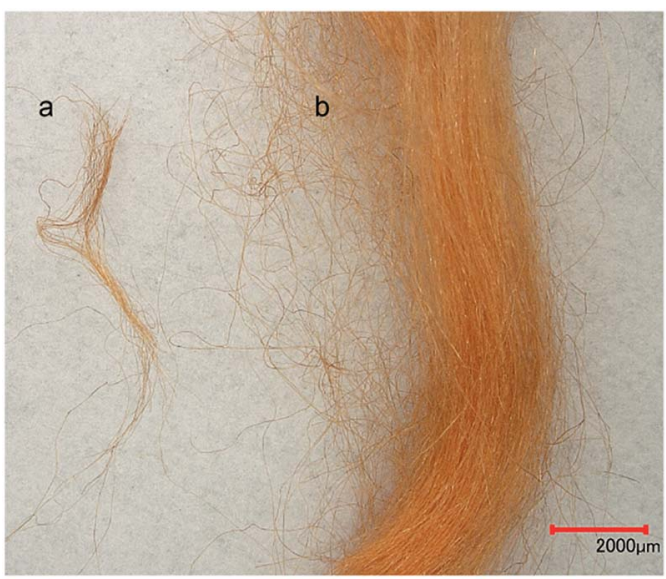

Fig. 3 Image of silk material dyed with madder: (a) aliquot $(100 \mu \mathrm{g})$ used for analysis by flowprobe TM MS (sample after measurements) and (b) material prior flowprobe ${ }^{\mathrm{TM}} \mathrm{MS}$ experiments. total reflection mode) spectroscopic, SEM (scanning electron microscopy), SEM-EDX (the combination of SEM with energy dispersive X-ray spectroscopy), HPLC-DAD (system 1), LC-ESIMS/MS (triple quadrupole, system 3 and 4) and ASAP-MS (capillary temperature: $150-250{ }^{\circ} \mathrm{C}$, capillary voltage: $100-$ $180 \mathrm{~V}$, source voltage: $10-20 \mathrm{~V}$, source voltage dynamic: 0-25 V, corona discharge: $5 \mu \mathrm{A}$, APCI source temperature: $250-400{ }^{\circ} \mathrm{C}$, positive and negative mode) studies were performed as previously reported. ${ }^{23,38}$ UV-Vis-spectra were measured in diffuse reflectance mode with a Cary 60 spectrophotometer from Agilent Technologies equipped with a remote diffuse reflection probe (Video Barrelino ${ }^{\mathrm{TM}}$ ) from Harrick Scientific (measurement range: $200-800 \mathrm{~nm}$; white standard: $\mathrm{BaSO}_{4}$ ).

The LMJ-SSP experiments were performed using a Prosolia flowprobe $^{\mathrm{TM}}$ system coupled to a QExactive ${ }^{\mathrm{TM}}$ Hybrid Quadrupole-Orbitrap mass spectrometer from Thermo Scientific (solvent for anthraquinones: methanol : water $(1: 1 \mathrm{v} / \mathrm{v})$ containing $0.1 \%(\mathrm{v} / \mathrm{v})$ formic acid or acetonitrile : water $(1: 1$ $\mathrm{v} / \mathrm{v}$ ) containing $0.1 \%(\mathrm{v} / \mathrm{v})$ formic acid, solvent for indigo-type dyes: methanol : water $(9: 1 \mathrm{v} / \mathrm{v})$ containing $0.2 \%(\mathrm{v} / \mathrm{v})$ formic acid and $1 \%(\mathrm{v} / \mathrm{v})$ tetrahydrofuran, solvent flow rate: $15-25 \mu \mathrm{L}$ $\min ^{-1}$, capillary temperature: $250{ }^{\circ} \mathrm{C}$, spray voltage: $4.5 \mathrm{kV}$ in positive mode and $3.0 \mathrm{kV}$ in negative mode, MS scan range: $200-$ $500 \mathrm{~m} / \mathrm{z}$ or $200-800 \mathrm{~m} / z$, resolution: 140000 , acquisition time: $2.2 \mathrm{~min}$, positive and negative mode). Genuine standard compounds were suspended or dissolved in methanol and spotted onto the surface of an Omni Slide ${ }^{\mathrm{TM}}$ (microscope glass slide from prosolia) by depositing a droplet of the suspension/ solution with following evaporation of the methanol. Single yarns or a bunch of fibers (historic samples: about 300-700 $\mu \mathrm{g}$ ) were placed to glass slides and wetted with a drop of the respective solvent for fixation (same solvent used for the in situ surface extraction). The slides were mounted into the xy movement stage of the flowprobe ${ }^{\mathrm{TM}}$ system and the nMotion software was used in manual mode for discrete analysis. Wash steps with spraying and data acquisition were performed between two samples to clean the flowprobe ${ }^{\mathrm{TM}}$ and to prevent carry-over of previous analytes.

The DESI experiments were carried out using a Prosolia Omni Spray ${ }^{\mathrm{TM}}$ ion source (different geometrical settings were tested: nebulisation capillary angle, nebulisation capillary-tosample distance, capillary inlet-to-sample distance and nebulisation capillary-to-capillary inlet distance was varied, nebulizing gas $\left(\mathrm{N}_{2}\right)$ pressure: 6 bar, solvent: methanol/water (different volume ratios) or acetonitrile/water (different volume ratios) containing formic acid or tetrahydrofuran in different concentrations, solvent flow rate: 1-10 $\mu \mathrm{L} \mathrm{min}^{-1}$, capillary temperature: $150-300{ }^{\circ} \mathrm{C}$, spray voltage: $3-5 \mathrm{kV}$, positive and negative mode). System coupled to a TSQ Quantum Ultra AM mass spectrometer from Thermo Scientific. Bunches of reference fibers were fixed onto glass slides with double-sided tape.

\subsection{Methods for extracting the colorants}

Blue fibers ${ }^{23}$ and red fibers ${ }^{38}$ were extracted as previously reported. Residual material (being insoluble in methanol or 
acetonitrile) from the extraction with formic acid-ethylenediaminetetraacetic acid was hydrolyzed with hydrochloric acid. $^{23}$

\subsection{Dyeing and mordanting procedure}

The vat dyeing process using synthetic indigo was performed as previously reported. ${ }^{23}$

Mordanting procedure of silken and woolen material (modified according to Cardon ${ }^{4}$ ):

Moistened fibers were treated for $20 \mathrm{~h}$ at $40{ }^{\circ} \mathrm{C}$ with an aqueous solution of $\mathrm{KAl}\left(\mathrm{SO}_{4}\right)_{2} \cdot 12 \mathrm{H}_{2} \mathrm{O}(0.5 \% \mathrm{~m} / \mathrm{v})$ and potassium bitartrate $(0.25 \% \mathrm{~m} / \mathrm{v})$. After cooling to room temperature the fibers were removed from the bath and rinsed with water. Moistened and stained fibers were used for the mordant dyeing procedure (modified according to Schweppe ${ }^{6}$ ) which is described below.

Mordant dyeing of silken and woolen material with food colorant E120:

The food colorant E120 (0.15 g) was dissolved in water (20 $\mathrm{mL}$ ). After the addition of the fibers, the dye bath was slowly heated to a temperature of $90^{\circ} \mathrm{C}$, and the temperature was kept at $70{ }^{\circ} \mathrm{C}$ for $1 \mathrm{~h}$. After cooling to room temperature the fibers were removed from the bath, rinsed with water and dried.

Mordant dyeing of silken and woolen material with dried rhizomes from madder:

A tea bag containing crushed rhizomes $(1 \mathrm{~g})$ was soaked for $17 \mathrm{~h}$ in water $(30 \mathrm{~mL})$. The extract was heated slowly within $1 \mathrm{~h}$ to beginning reflux, and the temperature was kept for an additional $10 \mathrm{~min}$. The rhizomes were removed, and the volume of the dye bath was filled up to a total volume of $100 \mathrm{~mL}$. The fibers were inserted and dyed for $30 \mathrm{~min}$ at $70{ }^{\circ} \mathrm{C}$ with occasional turning. After cooling to room temperature, the fibers were removed from the bath, rinsed with water and dried.

Mordant dyeing of silken material using either synthetic alizarin or purpurin:

To a suspension of the reference compound (approximately $50 \mathrm{mg})$ in water $(10 \mathrm{~mL}) 3$ drops of an aqueous $\mathrm{NH}_{4} \mathrm{OH}$ solution (25\% $\mathrm{NH}_{3}$ basis) were added and the silk were immersed and kept in the dye bath for $30 \mathrm{~min}$ at $70{ }^{\circ} \mathrm{C}$ with occasional turning. After cooling to room temperature, the fibers were removed from the bath, rinsed with water and dried.

\section{Conclusions}

Flowprobe ${ }^{\mathrm{TM}}$-ESI-HRMS was performed directly from the surface of various reference materials and archeological fibers, dyed with anthraquinone colorants gained from scale insects or plants of the Rubiaceae family and indigo-producing plants. This technique allowed the rapid analysis of different fiber materials under ambient conditions without any extra and timeconsuming sample preparation or a sophisticated sample fixation. This method is suitable to confirm the use of indigo-type and anthraquinone dyes - both dye classes which have been used for textile dyeing across all cultures and over all-time periods and for the rapid identification of the dye source. ESIMS detection proofed suitable for many organic dyestuffs and possesses the potential to expand the LMJ-SSP-MS technique to other dye compounds. The investigations were shown to be independent of fiber properties; thus, they are especially suitable for fragile archeological materials. The method allowed to examine bunches of fibers as well as single fragments of a yarn. Only a minimum amount of material is necessary, which remains intact during measurement, making this technology a minimal-invasive, reliable and time-saving application.

\section{Acknowledgements}

This work was supported by the German Federal Ministry of Education and Research (BMBF, grant number 01UO1310C). Samples from the finding site of Niya (Xinjiang Uyghur Autonomous Region, China) were taken within the framework of an interdisciplinary Chinese-German research project entitled "Silk Road Fashion - Clothes as a means of communication in the 1st millennium BC in Eastern Central Asia" and the Ancient Peruvian textiles (Ethnologisches Museum-Staatliche Museen zu Berlin) were sampled by Mrs L. Bjerregaard. The authors are grateful to the Archaeological Institute in Urumqi, and especially to Prof. Dr M. Wagner and Dr P. Wertmann (Deutsches Archaeologisches Institut) for continuing support. The authors would like to thank Mr U. Schwarzer (Landeskriminalamt Sachsen-Anhalt) for providing SEM- and SEM-EDX spectra, Dr C. Wagner and Prof. K. Merzweiler (Martin-Luther-Universität Halle-Wittenberg) for their allowance to use the ATR-IR- and UV/Vis-equipment, Dr M. Koch as well as Prof. W. Lorenz (Martin-Luther-Universität Halle-Wittenberg) for the allowance to use their LC-MS/MS equipment and Prof. C. Hertweck (Hans Knöll Institute, Jena) for the allowance to use the DESI-MSequipment. We also thank Dr K. Kania, Prof. Dr H. Hartmann (Technische Universität Dresden), Dr F.-K. Marcus (Sensient Food Colors, Geesthacht) and Mrs F. Hertel as well as Dr C.-H. Wunderlich (Landesamt für Denkmalpflege und Archäologie Sachsen-Anhalt - Landesmuseum für Vorgeschichte) for the donation of reference materials. Special thanks are due to Dr J. Franke (John Innes Centre, Norwich) for training in the utilization of the DESI-MS system and to Mr R. Gohlke (GSG Me $\beta$ und Analysengeräte $\mathrm{GmbH}$ ) for support.

\section{References}

1 M. P. Colombini, F. Modugno and E. Ribechini, in Mass Spectrometry Handbook, ed. M. S. Lee, John Wiley \& Sons, Inc., Hoboken, NJ, USA, 2012, pp. 797-828, DOI: 10.1002/ 9781118180730.ch36.

2 G. Spoto, in Mass Spectrometry Handbook, ed. M. S. Lee, John Wiley \& Sons, Inc., Hoboken, NJ, USA, 2012, pp. 845-858, DOI: $10.1002 / 9781118180730 . \operatorname{ch} 38$.

3 M. Gleba and U. Mannering, in Textiles and Textile Production in Europe: From Prehistory to $A D$ 400, ed. M. Gleba and U. Mannering, Oxbow Books, Oxford, 2012, pp. 1-24.

4 D. Cardon, Natural Dyes: Sources, Tradition, Technology and Science, Archetype Publications Ltd., London, 2007. 
5 J. H. Hofenk de Graaff, The Colourful Past: Origins, Chemistry and Identification of Natural Dyestuffs, Abegg-Stiftung and Archetype Publications Ltd., London, 2004.

6 H. Schweppe, Handbuch der Naturfarbstoffe: Vorkommen, Verwendung, Nachweis, ecomed, Landsberg/Lech, 1992.

7 M. Gulmini, A. Idone, E. Diana, D. Gastaldi, D. Vaudan and M. Aceto, Dyes Pigm., 2013, 98, 136-145.

8 A. Baran, A. Fiedler, H. Schulz and M. Baranska, Anal. Methods, 2010, 2, 1372-1376.

9 J. Lee, M.-H. Kim, K.-B. Lee, E. van Elslande, P. Walter and Y. Lee, Surf. Interface Anal., 2014, 46, 312-316.

10 I. Karapanagiotis, A. Lakka, L. Valianou and Y. Chryssoulakis, Microchim. Acta, 2008, 160, 477-483.

11 E. Rosenberg, Anal. Bioanal. Chem., 2008, 391, 33-57.

12 A. Serrano, M. M. Sousa, J. Hallett, J. A. Lopes and M. C. Oliveira, Anal. Bioanal. Chem., 2011, 401, 735-743.

13 J. Orska-Gawryś, I. Surowiec, J. Kehl, H. Rejniak, K. Urbaniak-Walczak and M. Trojanowicz, J. Chromatogr. A, 2003, 989, 239-248.

14 I. Petroviciu, F. Albu and A. Medvedovici, Microchem. J., 2010, 95, 247-254.

15 I. Degano, M. Biesaga, M. P. Colombini and M. Trojanowicz, J. Chromatogr. A, 2011, 1218, 5837-5847.

16 L. Taujenis and V. Olšauskaitè, Chemija, 2012, 23, 210-215.

17 L. G. Troalen, A. S. Phillips, D. A. Peggie, P. E. Barran and A. N. Hulme, Anal. Methods, 2014, 6, 8915-8923.

18 D. Mantzouris, I. Karapanagiotis, L. Valianou and C. Panayiotou, Anal. Bioanal. Chem., 2011, 399, 3065-3079.

19 N. Wyplosz, PhD thesis, University of Amsterdam, 2003.

20 Y. Lee, J. Lee, Y. Kim, S. Choi, S. W. Ham and K.-J. Kim, Appl. Surf. Sci., 2008, 255, 1033-1036.

21 C. Selvius DeRoo and R. A. Armitage, Anal. Chem., 2011, 83, 6924-6928.

22 C. J. Day, C. S. DeRoo and R. A. Armitage, in Archaeological chemistry VIII, ed. R. A. Armitage and J. H. Burton, American Chemical Society, Washington, DC, 2013, pp. 69-85.

23 A. Kramell, F. Porbeck, R. Kluge, A. Wiesner and R. Csuk, J. Mass Spectrom., 2015, 50, 1039-1043.

24 M. S. ElNaggar, B. Prideaux, V. Dartois and J. M. Wiseman, Curr. Metabolomics, 2014, 2, 122-131.

25 B. Prideaux, M. S. ElNaggar, M. Zimmerman, J. M. Wiseman, X. Li and V. Dartois, Int. J. Mass Spectrom., 2015, 377, 699708.

26 C.-C. Hsu, M. S. ElNaggar, Y. Peng, J. Fang, L. M. Sanchez, S. J. Mascuch, K. A. Møller, E. K. Alazzeh, J. Pikula, R. A. Quinn, Y. Zeng, B. E. Wolfe, R. J. Dutton, L. Gerwick, L. Zhang, X. Liu, M. Månsson and P. C. Dorrestein, Anal. Chem., 2013, 85, 7014-7018.

27 T. Gaissmaier, M. Siebenhaar, V. Todorova, V. Hullen and C. Hopf, Analyst, 2016, 141, 892-901.

28 V. Kertesz, M. J. Ford and G. J. Van Berkel, Anal. Chem., 2005, 77, 7183-7189.

29 V. Kertesz and G. J. Van Berkel, J. Mass Spectrom., 2010, 45, 252-260.
30 N. Talaty, C. C. Mulligan, D. R. Justes, A. U. Jackson, R. J. Noll and R. G. Cooks, Analyst, 2008, 133, 1532-1540.

31 D. R. Justes, N. Talaty, I. Cotte-Rodriguez and R. G. Cooks, Chem. Commun., 2007, 2142-2144, DOI: 10.1039/B703655H.

32 L. Valianou, I. Karapanagiotis and Y. Chryssoulakis, Anal. Bioanal. Chem., 2009, 395, 2175-2189.

33 K. K. A. de Mayolo, Econ. Bot., 1989, 43, 181-191.

34 X. Zhang, I. Good and R. Laursen, J. Archaeol. Sci., 2008, 35, 1095-1103.

35 J. Liu, D. Guo, Y. Zhou, Z. Wu, W. Li, F. Zhao and X. Zheng, J. Archaeol. Sci., 2011, 38, 1763-1770.

36 Y. Xie, Y. Xiong, Y. Chen, L. Hu, M. Yin and Z. Chen, Sciences of conservation \& archaeology, 2001, 1, 1-7.

37 J. H. Hofenk de Graaff and M. van Bommel, in Fabulous creatures from the desert sands: Central Asian woolen textiles from the second century $B C$ to the second century $A D$, ed. $\mathrm{D}$. Keller and R. Schorta, Abegg-Stiftung, Riggisberg, 2001, vol. 10.

38 A. Kramell, X. Li, R. Csuk, M. Wagner, T. Goslar, P. E. Tarasov, N. Kreusel, R. Kluge and C.-H. Wunderlich, Quat. Int., 2014, 348, 214-223.

39 J. Liu, C. Mouri, R. Laursen, F. Zhao, Y. Zhou and W. Li, J. Archaeol. Sci., 2013, 40, 4444-4449.

$40 \mathrm{H}$. Farke, Archäologische Fasern, Geflechte, Gewebe: Bestimmung und Konservierung, Museum für Ur- und Frühgeschichte Thüringens, Weimar, 1986.

41 K. Lech, K. Witkoś, B. Wileńska and M. Jarosz, Anal. Bioanal. Chem., 2015, 407, 855-867.

42 K. Stathopoulou, L. Valianou, A.-L. Skaltsounis, I. Karapanagiotis and P. Magiatis, Anal. Chim. Acta, 2013, 804, 264-272.

43 H. Itokawa, Y. Qiao and K. Takeya, Phytochemistry, 1989, 28, 3465-3468.

44 C. Mouri and R. Laursen, Microchim. Acta, 2012, 179, 105113.

45 A. E. Kramell, P. Wertmann, D. Hosner, R. Kluge, F. Oehler, C.-H. Wunderlich, P. E. Tarasov, M. Wagner and R. Csuk, J. Archaeol. Sci. Reports, 2016, 10, 464-473.

46 I. Vanden Berghe, M. Gleba and U. Mannering, J. Archaeol. Sci., 2009, 36, 1910-1921.

47 R. Hofmann-de Keijzer, M. R. van Bommel and I. Joosten, in Hallstatt Textiles: Technical Analysis, Scientific Investigation and Experiment on Iron Age Textiles, ed. P. Bichler, $\mathrm{K}$. Grömer, R. Hofmann-de Keijzer, A. Kern and $H$. Reschreiter, Archaeopress, Oxford, 2005, vol. BAR International Series 1351, pp. 55-72.

48 G. G. Balakina, V. G. Vasiliev, E. V. Karpova and V. I. Mamatyuk, Dyes Pigm., 2006, 71, 54-60.

49 I. Degano and M. P. Colombini, J. Archaeol. Sci., 2009, 36, 1783-1790.

50 V. Kertesz and G. J. Van Berkel, Anal. Chem., 2010, 82, 59175921.

51 T. Takano, T. Kondo and F. Nakatsubo, J. Wood Sci., 2006, 52, 90-92. 